\title{
Simultaneous Determination of Menaquinone-4 and Its Metabolite in Human Osteoblasts by High-Performance Liquid Chromatography/Atmospheric Pressure Chemical Ionization Tandem Mass Spectrometry
}

\author{
Yoshihisa SANo*†, Kiyomi KIKUCHI*, Kyoichi TaDANo*, Kazuko Hoshi** \\ and Yasuko KoshiHaRA** \\ *Tsukuba Research Laboratories, Eisai Co., Ltd., Tsukuba, Ibaraki 300-26, Japan \\ ** Department of Biosignal Research, Tokyo Metropolitan Institute of Gerontology, \\ Sakaecho, Itabashi, Tokyo 173, Japan
}

\begin{abstract}
A simple and sensitive method is described for the determination of menaquinone- 4 and its metabolite menaquinone-4 epoxide in human periosteal osteoblasts. The method is based on high-performance liquid chromatography/ atmospheric pressure chemical ionization mass spectrometry, using deuterated menaquinone-4 as an internal standard. Selected reaction monitoring (SRM) is employed for selectivity and sensitivity. This enables quantification in a short period of time (within $5 \mathrm{~min}$ ) over the extended ranges $1-1000 \mathrm{ng} / \mathrm{ml}$ for menaquinone-4 and $2.5-5000 \mathrm{ng} / \mathrm{ml}$ for menaquinone-4 epoxide with acceptable precision and accuracy. The method was used to determine both compounds in human osteoblasts, following incubation with menaquinone-4. It is suggested that menaquinone-4 is metabolized to menaquinone-4 epoxide in bone as well as in liver, indicating the drug has the same role in $\gamma$-carboxylation of vitamin $\mathrm{K}$ dependent proteins in both tissues. It is expected that this method will contribute to elucidating the pharmacological mechanism of action of menaquinone- 4 on bone metabolism.
\end{abstract}

Keywords Menaquinone-4, vitamin K, mass spectrometry, osteoblast, metabolism, $\gamma$-carboxylase

Menaquinone-4 (vitamin $\mathbf{K}_{2}$ ) is a potent cofactor for $\gamma$-carboxylase, which was originally observed in liver. This enzyme plays an essential role in $\gamma$-carboxylation of gla( $\gamma$-carboxy glutamic acid)-containing proteins of the bone matrix, such as osteocalcin. In bone, $\gamma$-carboxylated osteocalcin is known to be involved in the bone remodeling system ${ }^{1-3}$, and the pharmacological activity of menaquinone- 4 has been demonstrated in human osteoblasts in vitro ${ }^{4-6}$ and also in ovariectomized rats, the latter serving as an in vivo model for human osteoporosis. ${ }^{7}$ Menaquinone- 4 is now available as a medication for the treatment of osteoporosis. ${ }^{8}$

The pharmacokinetic profile of menaquinone-4 in rodents and dogs has already been reported. ${ }^{9-14}$ The specific distribution of menaquinone- 4 into bone, the target tissue of the compound, after oral dosing, has also been demonstrated in dogs and ovariectomized rats. ${ }^{15-17}$ The metabolism of menaquinone- 4 in bone following distribution has not been elucidated, although the metabolism is thought to be similar in bone and liver. The latter recycles vitamin K via its epoxide for the $\gamma$ carboxylation of vitamin $\mathrm{K}$ dependent proteins such as

$\dagger$ To whom correspondence should be addressed. blood coagulation factors.

In this study, we developed a simultaneous assay method for menaquinone-4 and its metabolite menaquinone4 epoxide in human osteoblasts, where bone formation actively takes place, using high-performance liquid chromatography (HPLC)/atmospheric pressure chemical ionization tandem mass spectrometry. The method was used to determine both compounds in human osteoblasts following incubation with menaquinone-4 for $10 \mathrm{~d}$. The metabolic profile of menaquinone- 4 in bone is described for the first time.

\section{Experimental}

\section{Chemicals and reagents}

Menaquinone-4 (2-methyl-3-tetraprenyl-1,4-naphthoquinone) was synthesized by Eisai Chemical Co., Ltd. (Ibaraki, Japan). $\left[{ }^{2} \mathrm{H}_{7}\right]$ menaquinone-4 (deuterated menaquinone-4) was synthesized and supplied by Huntingdon Life Sciences (Cambridgeshire, UK). The isotopic purity of deuterated menaquinone- 4 was $98.5 \%$. Menaquinone-4 2,3-epoxide was synthesized by Eisai Co., Ltd. (Tokyo, Japan). The structures of these compounds are 
<smiles>CCOC(=O)c1ccccc1</smiles>

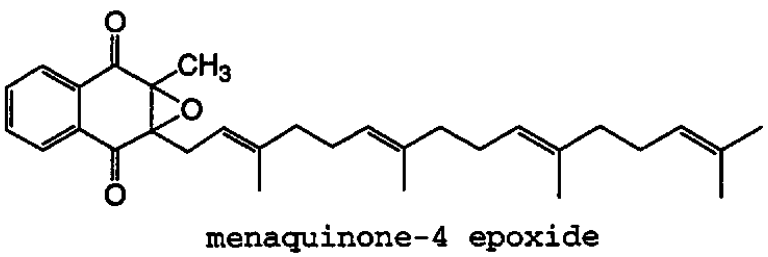<smiles>[2H]c1c([2H])c([2H])c2c(c1[2H])C(=O)C(C/C=C(\C)CC/C=C(\C)CC/C=C(\C)CCC=C(C)C)=C(C([2H])([2H])[18O])C2=O</smiles>

Fig. 1 Chemical structures of menaquinone-4, menaquinone4 epoxide and $\left[{ }^{2} \mathrm{H}_{7}\right]$ menaquinone- 4 (deuterated menaquinone-4).

shown in Fig. 1. Solvents for HPLC were of HPLC grade obtained from Wako Pure Chemical Industries, Ltd. (Osaka, Japan). Fetal bovine serum (FBS) and $\alpha$ minimum essential medium ( $\alpha$-MEM) were purchased from Irvine Scientific (Santa Ana, CA, USA). Disodium $\alpha$-glycerophosphate ( $\alpha$-GP) was purchased from Tokyo Kasei Co. (Tokyo, Japan). Warfarin was obtained from Eisai Co., Ltd. (Tokyo, Japan).

\section{Apparatus for LC/APCI/MS/MS}

HPLC was performed on an ODS column (L-column ODS, $150 \mathrm{~mm} \times 1.5 \mathrm{~mm}$ i.d., Chemicals Inspection and Institute, Tokyo, Japan) using a Hewlett-Packard HP 1050 series system (Waldbronn, Germany). The mobile phase was EtOH/MeOH $(80: 20)$ with a flow rate of $0.15 \mathrm{ml} / \mathrm{min}$.

Mass spectrometric detection was carried out with a Finnigan TSQ 7000 triple-quadrupole instrument (San Jose, CA, USA) using positive ion atmospheric pressure chemical ionization (APCI) as an LC/MS interface. The temperatures of the vaporizer and heated capillary were set at $400^{\circ} \mathrm{C}$ and $125^{\circ} \mathrm{C}$, respectively. The sheath gas pressure and auxiliary gas flow were set at $65 \mathrm{psi}$ and 5 units, respectively. Selected reaction monitoring (SRM) was employed using argon as the collision gas at $2 \mathrm{mTorr}$, with collision energies of $-25 \mathrm{eV}$ for menaquinone- 4 and deuterated menaquinone- 4 and $-12.5 \mathrm{eV}$ for menaquinone- 4 epoxide during collisioninduced dissociation (CID). Parent to daughter transitions were monitored for $m / z 445$ to $m / z \quad 187$ for menaquinone-4, $m / z 461$ to $m / z 311$ for menaquinone-4 epoxide and $m / z 452$ to $m / z \quad 194$ for deuterated menaquinone-4. Total scan time was set at $2 \mathrm{~s}$.

\section{Preparation of calibration curve}

The stock solutions of menaquinone- 4 and menaquinone-4 2,3-epoxide (both $0.5 \mathrm{mg} / \mathrm{ml}$ ) were prepared in methanol and stored at $-20^{\circ} \mathrm{C}$. Standard solutions for the calibration curves in each analysis were prepared by sequential dilution of the stock solution with methanol (final concentrations of 1, 2.5, 5, 10, 50, 100, 500,1000 and $5000 \mathrm{ng} / \mathrm{ml}$ ) and by mixing with an internal standard, deuterated menaquinone- $4(100 \mathrm{ng} / \mathrm{ml}$ as a final concentration). Twenty-microliter aliquots of these samples were subjected to LC/APCI/MS/MS analysis. QC samples $(5,50$ and $500 \mathrm{ng} / \mathrm{ml}$ in duplicate) were also prepared by adding the appropriate concentrations of standard solutions and deuterated menaquinone-4 $(100 \mathrm{ng} / \mathrm{ml}$ as a final concentration) to $1 \mathrm{ml}$ blank samples (cell extract). The reliability of the measurement was confirmed using these QC samples.

\section{Quantification method}

Calibration curves were constructed by plotting the peak area ratios of the analytes (menaquinone-4 and menaquinone-4 epoxide) to the internal standard (deuterated menaquinone-4) against the concentration of analytes. A weighted $\left(1 / X^{2}\right)$ linear regression line was fitted over the 1000-fold concentration range. The concentrations of menaquinone-4 and menaquinone- 4 epoxide in test samples were calculated from this line and are expressed as $\mathrm{ng} / \mathrm{ml}$ or $\mathrm{ng} /$ well.

\section{Incubation of human osteoblasts with menaquinone-4}

Human periosteal osteoblasts were incubated with menaquinone- 4 to investigate the metabolism of the compound. The isolation and cell culture methods have been described previously. ${ }^{5,18}$ Incubation media consisted of $\alpha$-MEM supplemented with $10 \%$ FBS containing menaquinone-4 $\left(2.5 \times 10^{-6} \mathrm{M}\right)$ with or without warfarin $\left(2.5 \times 10^{-5} \mathrm{M}\right)$. Cells at 18 th population doubling levels in 12-well multi-well plates $\left(4 \mathrm{~cm}^{2} /\right.$ well) that had reached confluence were incubated in media at $37^{\circ} \mathrm{C}$ in $5 \% \mathrm{CO}_{2} / 95 \%$ air for $10 \mathrm{~d}$ in the presence of $2 \mathrm{mM} \alpha$-GP, as $10 \mathrm{mM} \beta$-GP, to accelerate the mineralization process. Menaquinone-4 and warfarin were dissolved in dimethyl sulfoxide (DMSO) and water, respectively. The final concentration of DMSO was less than $0.1 \%$; the same amount of DMSO was added to the control culture. Media were changed every other day.

\section{Sample preparation}

The media was removed after incubation, the cells were washed twice with $1.5 \mathrm{ml}$ phosphate buffered saline $\left(\mathrm{Ca}^{2+}\right.$ and $\mathrm{Mg}^{2+}$ free) containing $1 \% \mathrm{DMSO}$ and extracted with $1.5 \mathrm{ml}$ methanol. Deuterated menaquinone-4 (final concentration of $100 \mathrm{ng} / \mathrm{ml}$ ) was added to the extract and $20 \mu \mathrm{l}$ aliquots were subjected to LC/APCI/MS/MS analysis. 

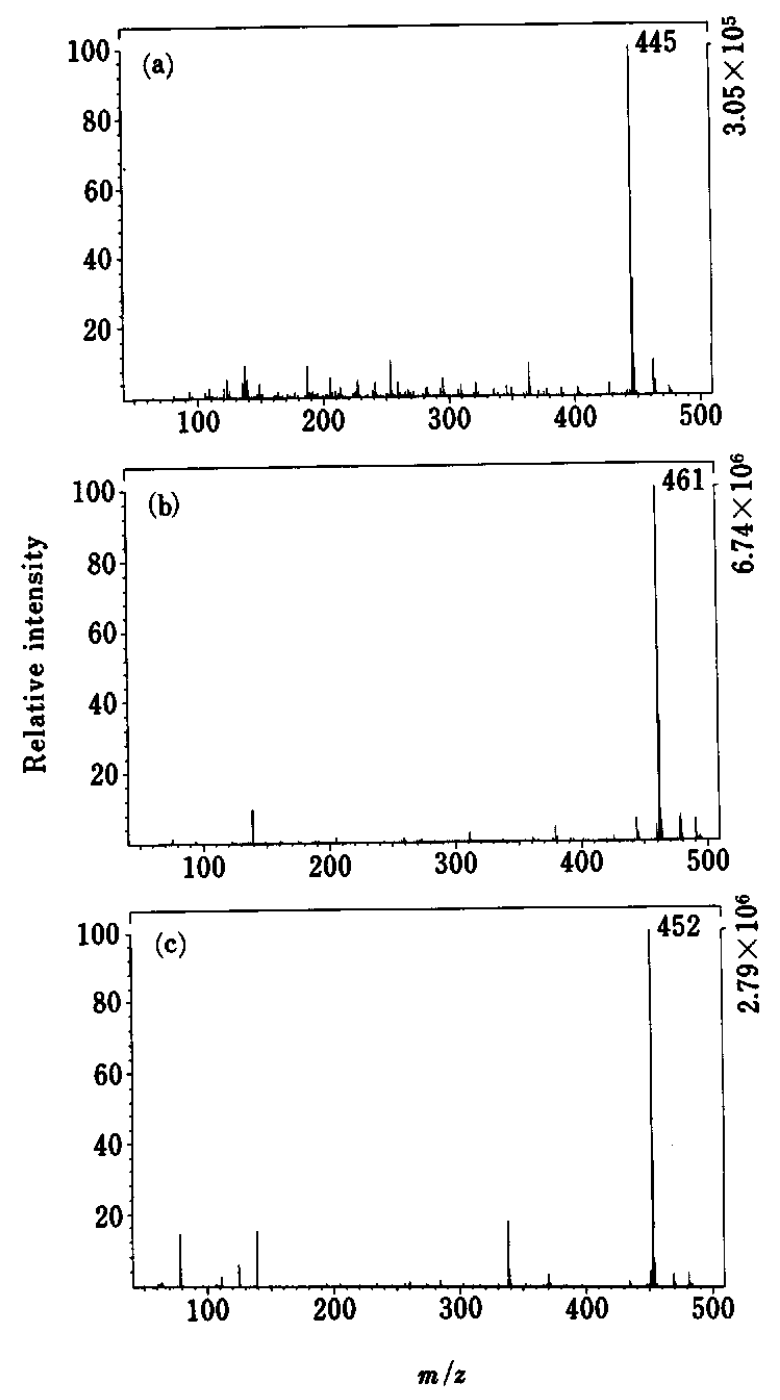

Fig. 2 APCI positive mass spectra of menaquinone-4 (a), menaquinone-4 epoxide (b) and deuterated menaquinone-4 (c).

\section{Results and Discussion}

\section{LC/MS/MS conditions}

First we attempted to raise the intensity of the protonated molecular ions of menaquinone-4 and its metabolite on LC/MS to achieve high sensitivity detection. Figure 2 shows the APCI positive mass spectra for menaquinone-4, menaquinone-4 epoxide and deuterated menaquinone-4. The protonated molecular ions for these compounds were observed as base peak ions under these conditions. A preliminary study revealed that electrospray ionization (ESI) positive mass spectra of menaquinone-4 indicated a mixture of some adduct ions to the molecular ion, including $\mathrm{Na}$ adduct ions, and they were not altered in any component of the mobile phase and at any heated capillary temperature examined (data not shown). We also observed that highly aqueous mobile phases, such as $50 \%$ aqueous methanol, result in the simple protonated molecular ion $(m / z 445)$ instead

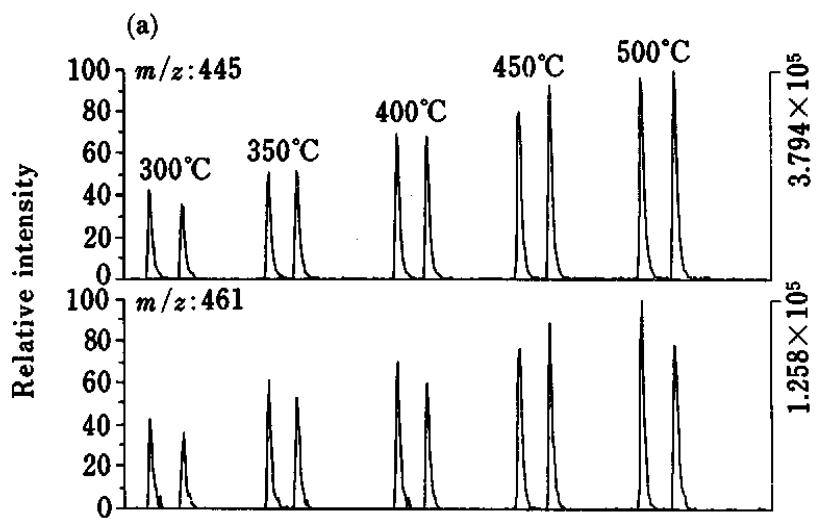

(b)

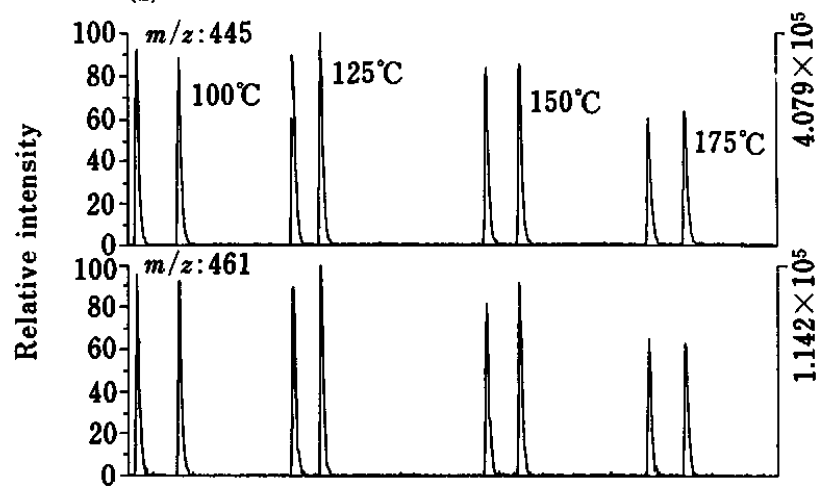

Fig. 3 The effects of vaporizer (a) and heated capillary (b) temperatures on the intensity of the protonated ions of menaquinone-4 and menaquinone-4 epoxide.

of these adduct ions, although menaquinone-4 was not eluted from the HPLC column under such aqueous conditions. The weak intensity of the individual adduct ions was expected to result in low sensitivity on LC/MS/ MS analysis. In general, $\mathrm{Na}$ adduct ions cannot be fragmented during subsequent CID to obtain an appropriate product ion. We concluded therefore that ESI is not suitable for LC/MS/MS analysis of menaquinone-4 or its metabolite, while APCI, in which any component of the HPLC mobile phase can generate the protonated molecular ions for the parent ions on LC/MS/MS analysis, is suitable as an LC/MS interface.

For the HPLC mobile phase, we found that organic solvents and salts such as ammonium acetate did not affect peak shape or the intensity of menaquinone-4. Ethanol/methanol $(80: 20)$ was selected as the mobile phase since it can separate the analytes from each other within a short time (5 min) on HPLC chromatograms.

Figure 3 shows the effects of vaporizer (a) and heated capillary (b) temperatures on the intensity of the protonated molecular ions of menaquinone- 4 and menaquinone-4 epoxide. It can be seen that the intensity is quite dependent on these two parameters. Both temperatures also affected the background levels on chromatograms (data not shown). The vaporizer and heated capillary temperatures were set at $400^{\circ} \mathrm{C}$ and 

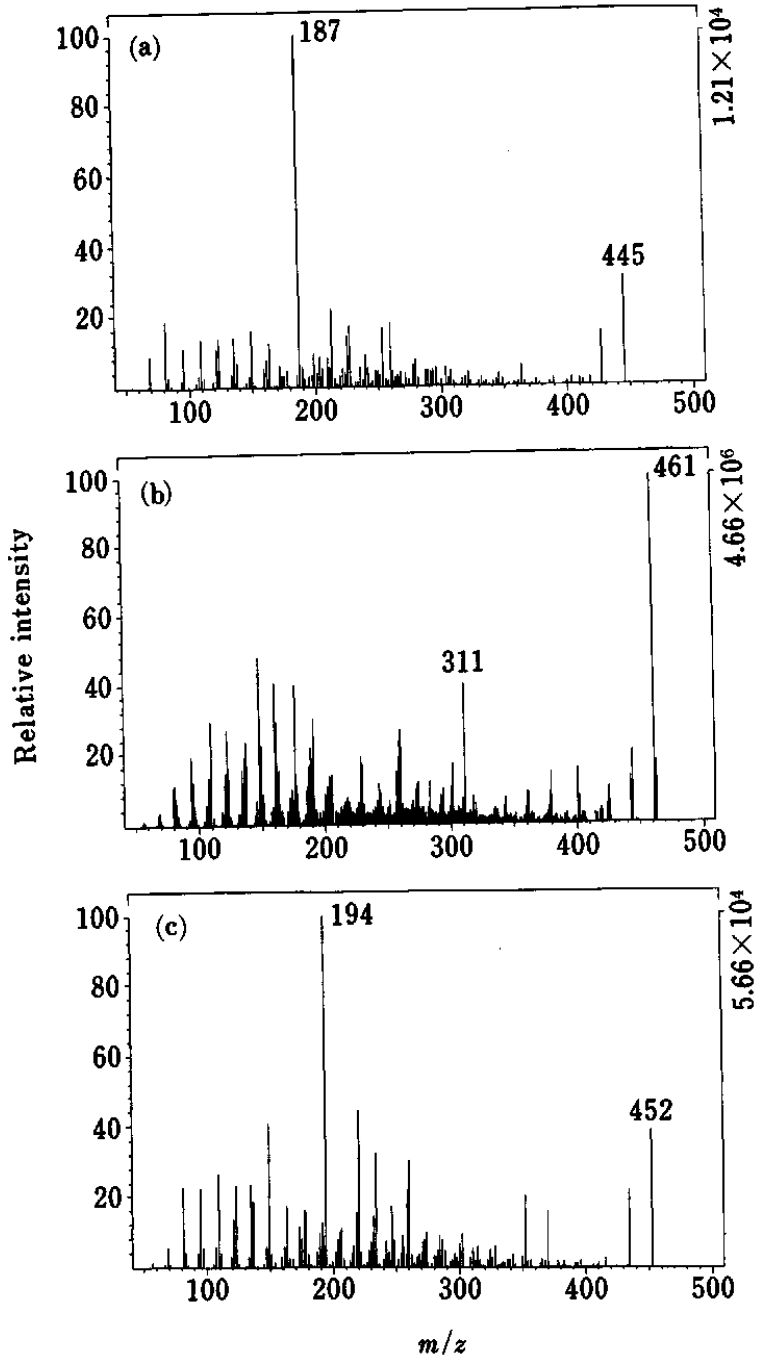

Fig. 4 Product ion mass spectra of the protonated ions of menaquinone-4 (a), menaquinone-4 epoxide (b) and deuterated menaquinone-4 (c).

$125^{\circ} \mathrm{C}$, respectively, taking into consideration the signalto-noise ratio of the peak intensity. The sheath gas pressure and auxiliary gas flow did not greatly affect the sensitivities of these compounds. Under the conditions mentioned above, menaquinone- 4 and its metabolite produced the protonated molecule the most effectively with no apparent fragmentation.

We decided to use selected reaction monitoring (SRM) for the determination of these compounds to achieve high selectivity and sensitivity. Figure 4 shows the product ion mass spectra of the protonated ions of menaquinone4, menaquinone- 4 epoxide and deuterated menaquinone4. Fragmentation of the parent ions is apparent, with $m / z 187,311$ and 194 being prominent product ions for the three compounds, respectively. The effects of the collision energy and collision gas pressure on the intensity were investigated (data not shown) in order to obtain a maximum response of the product ions. Based on this result, the energy level was set at $-25 \mathrm{eV}$ for

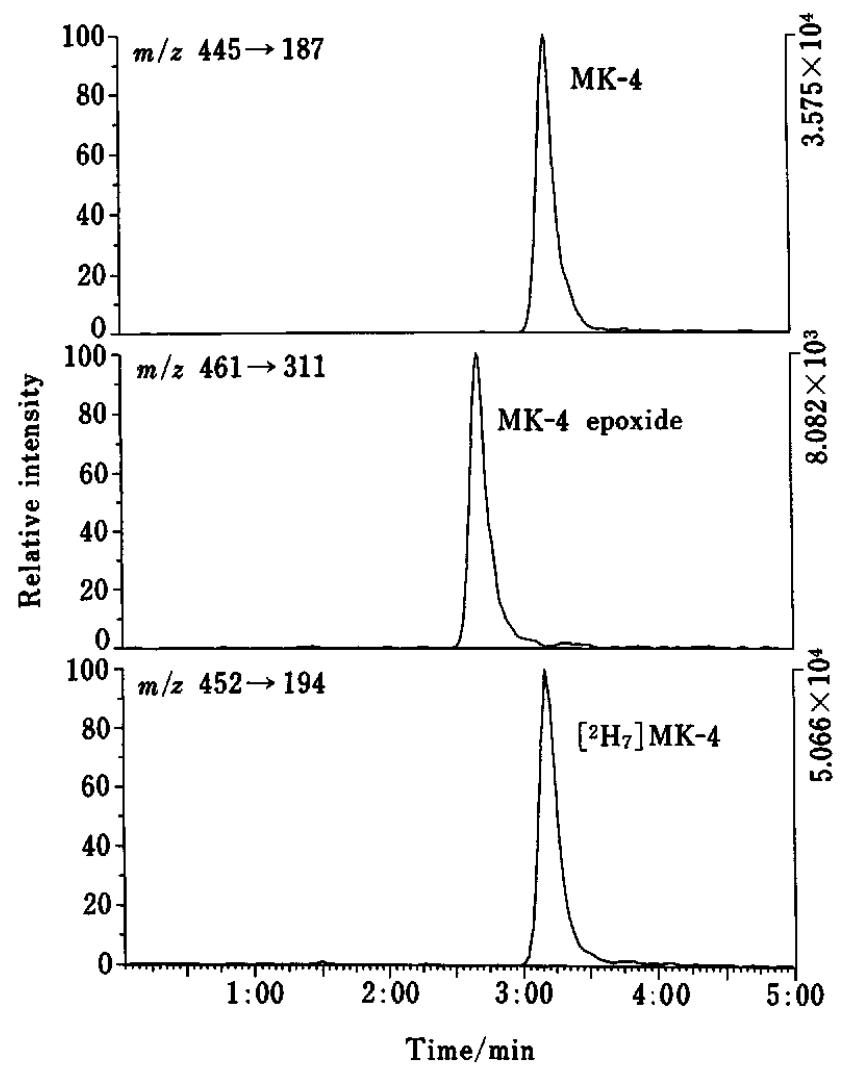

Fig. 5 Typical LC/APCI/MS/MS chromatograms for menaquinone-4, menaquinone-4 epoxide and deuterated menaquinone-4. MK-4: menaquinone-4; MK-4 epoxide: menaquinone-4 epoxide.

menaquinone-4 and deuterated menaquinone- 4 and $-12.5 \mathrm{eV}$ for menaquinone- 4 epoxide. The argon gas pressure was $2 \mathrm{mTorr}$ for both.

\section{Calibration curves}

Figure 5 presents typical LC/APCI/MS/MS chromatograms for menaquinone-4, menaquinone-4 epoxide and deuterated menaquinone-4 obtained from the calibration samples. It should be noted that menaquinone4 and menaquinone- 4 epoxide were sufficiently separated from each other in a short period of time (within $5 \mathrm{~min}$ ) and the respective chromatograms of the compounds did not interfere with each other. No interfering peak appeared at the retention time of the two compounds on the chromatogram of the blank sample. Figure 6 shows typical calibration curves for menaquinone-4 and menaquinone- 4 epoxide. Responses in each analysis were linear over the range from 1 to $1000 \mathrm{ng} / \mathrm{ml}$ for menaquinone-4 $(r \geq 0.996)$ and from 2.5 to $5000 \mathrm{ng} / \mathrm{ml}$ for menaquinone-4 epoxide ( $r \geq 0.996$ ), indicating satisfactory concentration ranges with which to determine the compounds in biological samples.

\section{Precision and accuracy}

The accuracy and intra- and inter-day precision were determined for the present assay method. The results 
(a)

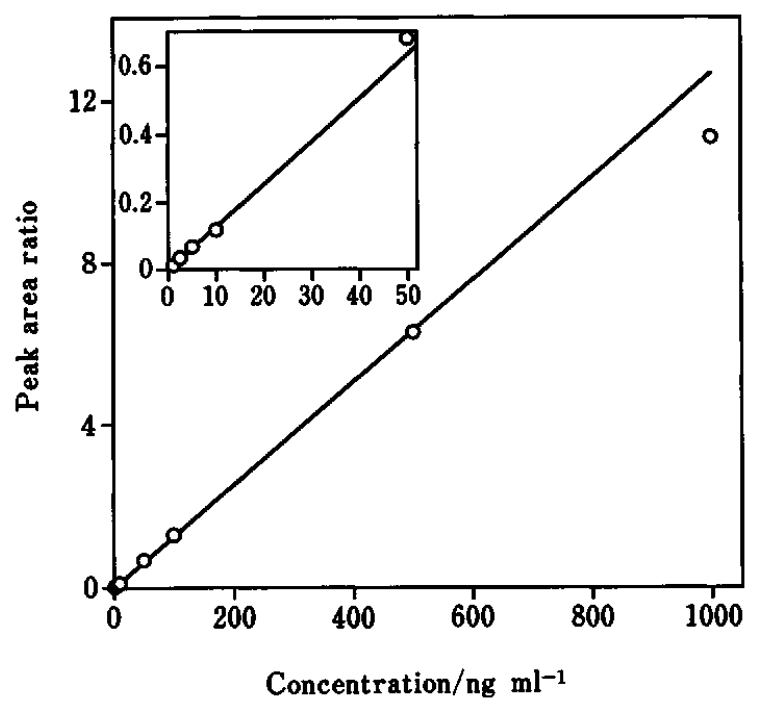

(b)

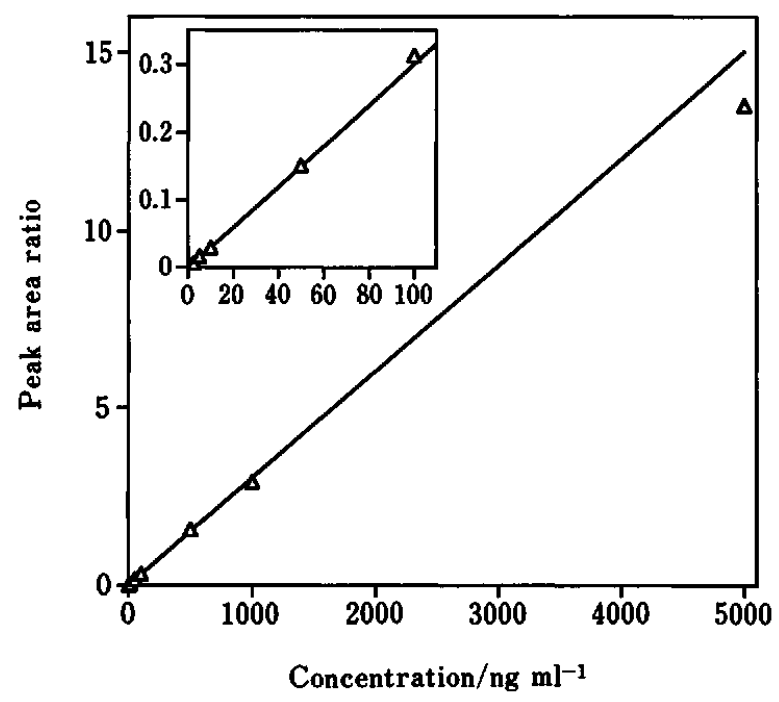

Fig. 6 Typical calibration curves for menaquinone-4 (a) and menaquinone-4 epoxide (b). Menaquinone-4: $1-1000 \mathrm{ng} / \mathrm{ml}(r=0.9966)$; menaquinone-4 epoxide: $2.5-5000 \mathrm{ng} / \mathrm{ml}(r=0.9975)$. The plots over the short range are shown in the insets.

Table 1 The accuracy and intra- and inter-day precision for menaquinone-4 and menaquinone-4 epoxide in the calibration samples

\begin{tabular}{|c|c|c|c|c|c|c|c|}
\hline \multirow[b]{2}{*}{$\begin{array}{c}\text { Nominal } \\
\text { concentration/ } \\
\mathrm{ng} \mathrm{ml}^{-1}\end{array}$} & \multirow[b]{2}{*}{$\begin{array}{c}\text { Injection } \\
\text { amount/ } \\
\text { pg }\end{array}$} & \multicolumn{3}{|c|}{$\mathrm{MK}-4$} & \multicolumn{3}{|c|}{ MK-4 epoxide } \\
\hline & & $\begin{array}{c}\text { Accuracy, } \\
\% \\
{[n=5]}\end{array}$ & $\begin{array}{c}\text { Intra-day } \\
\text { precision } \\
\text { (RSD, \%) } \\
{[n=5]}\end{array}$ & $\begin{array}{c}\text { Inter-day } \\
\text { precision } \\
\text { (RSD, \%) } \\
{[n=3]}\end{array}$ & $\begin{array}{c}\text { Accuracy, } \\
\% \\
{[n=5]}\end{array}$ & $\begin{array}{c}\text { Intra-day } \\
\text { precision } \\
\text { (RSD, \%) } \\
{[n=5]}\end{array}$ & $\begin{array}{c}\text { Inter-day } \\
\text { precision } \\
\text { (RSD, \%) } \\
{[n=3]}\end{array}$ \\
\hline 1 & 20 & -1.00 & 6.06 & 5.05 & & & \\
\hline 2.5 & 50 & 4.00 & 9.62 & 10.89 & -0.40 & 13.65 & 4.12 \\
\hline 5 & 100 & -2.20 & 4.09 & 5.60 & 2.60 & 6.63 & 7.82 \\
\hline 10 & 200 & 2.60 & 1.36 & 8.02 & -4.40 & 5.23 & 1.48 \\
\hline 50 & 1000 & 3.28 & 1.37 & 1.45 & 0.70 & 2.48 & 1.80 \\
\hline 100 & 2000 & 2.64 & 2.13 & 2.06 & 0.27 & 3.49 & 2.37 \\
\hline 500 & 10000 & -1.22 & 1.29 & 3.00 & 6.50 & 1.26 & 3.79 \\
\hline 1000 & 20000 & -7.61 & 1.25 & 3.97 & 3.26 & 1.47 & 0.18 \\
\hline 5000 & 100000 & & & & -8.64 & 0.97 & 3.14 \\
\hline
\end{tabular}

MK-4: menaquinone-4; MK-4 epoxide: menaquinone-4 epoxide.

Table 2 The accuracy and inter-day precision for menaquinone-4 and menaquinone-4 epoxide in QC samples

\begin{tabular}{|c|c|c|c|c|c|}
\hline \multirow{2}{*}{$\begin{array}{c}\text { Nominal } \\
\text { concentration/ } \\
\mathrm{ng} \mathrm{ml}^{-1}\end{array}$} & \multirow{2}{*}{$\begin{array}{c}\text { Injection } \\
\text { amount/ } \\
\text { pg }\end{array}$} & \multicolumn{2}{|c|}{ MK-4 } & \multicolumn{2}{|c|}{ MK-4 epoxide } \\
\hline & & $\begin{array}{c}\text { Accuracy, } \\
\% \\
{[n=3]}\end{array}$ & $\begin{array}{c}\text { Precision } \\
\text { (RSD, \%) } \\
{[n=3]}\end{array}$ & $\begin{array}{c}\text { Accuracy, } \\
\% \\
{[n=3]}\end{array}$ & $\begin{array}{c}\text { Precision } \\
\text { (RSD, \%) } \\
{[n=3]}\end{array}$ \\
\hline 5 & 100 & 1.80 & 4.13 & -3.60 & 8.92 \\
\hline 5 & 100 & -1.00 & 4.04 & -2.40 & 11.27 \\
\hline 50 & 1000 & 6.18 & 3.99 & 9.44 & 1.96 \\
\hline 50 & 1000 & 6.30 & 2.99 & 6.82 & 3.05 \\
\hline 500 & 10000 & -6.99 & 2.29 & -0.43 & 0.73 \\
\hline 500 & 10000 & -11.12 & 2.86 & -4.69 & 1.81 \\
\hline
\end{tabular}

MK-4: menaquinone-4; MK-4 epoxide: menaquinone-4 epoxide. 
Table 3 The concentrations of menaquinone-4 and menaquinone-4 epoxide in osteoblasts incubated with menaquinone-4 $\left(2.5 \times 10^{-6} \mathrm{M}\right)$ with or without warfarin $\left(2.5 \times 10^{-5} \mathrm{M}\right)$ for $10 \mathrm{~d}$

\begin{tabular}{lccc}
\hline \multirow{2}{*}{ Incubated compound } & \multicolumn{2}{c}{ Found concentration } & $\begin{array}{c}\text { Ratio } \\
\text { (Epo/MK-4) }\end{array}$ \\
\cline { 2 - 4 } & $\begin{array}{c}\text { Menaquinone-4 } \\
(\mathrm{ng} / \text { well) }\end{array}$ & $\begin{array}{c}\text { Menaquinone-4 epoxide } \\
\text { (ng/well) }\end{array}$ & 1.41 \\
Menaquinone-4 & 8.66 & 12.20 & 2.79 \\
\hline
\end{tabular}

Epo: menaquinone-4 epoxide; MK-4: menaquinone-4. Each value represents the mean of 4 samples.

are shown in Table 1. The accuracy (mean of 5 replicates) for both menaquinone-4 and menaquinone-4 epoxide was satisfactory (within $\pm 8.64 \%$ over the calibration range). The intra- and inter-day precision (5 and 3 replicates, respectively) were also acceptable for both compounds. The RSD values were generally less than $10 \%$, except for the intra-day precision of the lowest menaquinone-4 epoxide concentration, which was $13.65 \%$. Based on these results, we concluded that the quantification limits for menaquinone- 4 and menaquinone-4 epoxide were $1 \mathrm{ng} / \mathrm{ml}$ ( $20 \mathrm{pg}$ per injection) and $2.5 \mathrm{ng} / \mathrm{ml}$ ( $50 \mathrm{pg}$ per injection), respectively.

To evaluate the effects of endogenous interferences extracted from osteoblasts on mass chromatograms, QC samples were prepared by adding menaquinone- 4 and menaquinone- 4 epoxide to the cell extracts at concentrations of 5,50 and $500 \mathrm{ng} / \mathrm{ml}$. These mixtures were then subjected to LC/MS/MS analysis. The results are presented in Table 2. The accuracy of all six QC samples ( 2 lots at 3 concentrations) was within $\pm 11.12 \%$ (mean of triplicates). This result is acceptable and indicates that interfering peaks on the chromatograms are negligible. The present LC/MS/MS assay method therefore is thought to be sufficiently reliable for measuring menaquinone- 4 and menaquinone -4 epoxide in samples extracted from osteoblasts.

\section{Measurement of menaquinone-4 and menaquinone-4 epoxide in osteoblasts}

The method has been used to determine menaquinone4 and menaquinone-4 epoxide in human osteoblasts following incubation with menaquinone- $4\left(2.5 \times 10^{-6} \mathrm{M}\right)$ for $10 \mathrm{~d}$ with or without warfarin $\left(2.5 \times 10^{-5} \mathrm{M}\right)$ in order to investigate the metabolism of the compound in bone. The concentrations for all of the control samples, to which no menaquinone-4 was added before incubation, were below the quantification limit for both compounds, confirming that endogenous compounds do not interfere with the measurement of menaquinone-4 and menaquinone-4 epoxide in osteoblasts. It has been reported $^{1-3}$ that vitamin $\mathrm{K}$ (quinone form) was metabolized to its epoxide via the hydroquinone form, and then converted to the quinone form by the enzyme vitamin $\mathbf{K}$ epoxide reductase in liver. Warfarin is thought to inhibit the enzyme, and the epoxide increased when the liver was exposed to the drug. ${ }^{1-3}$ In the present study, menaquinone-4 epoxide was detected in cell extracts

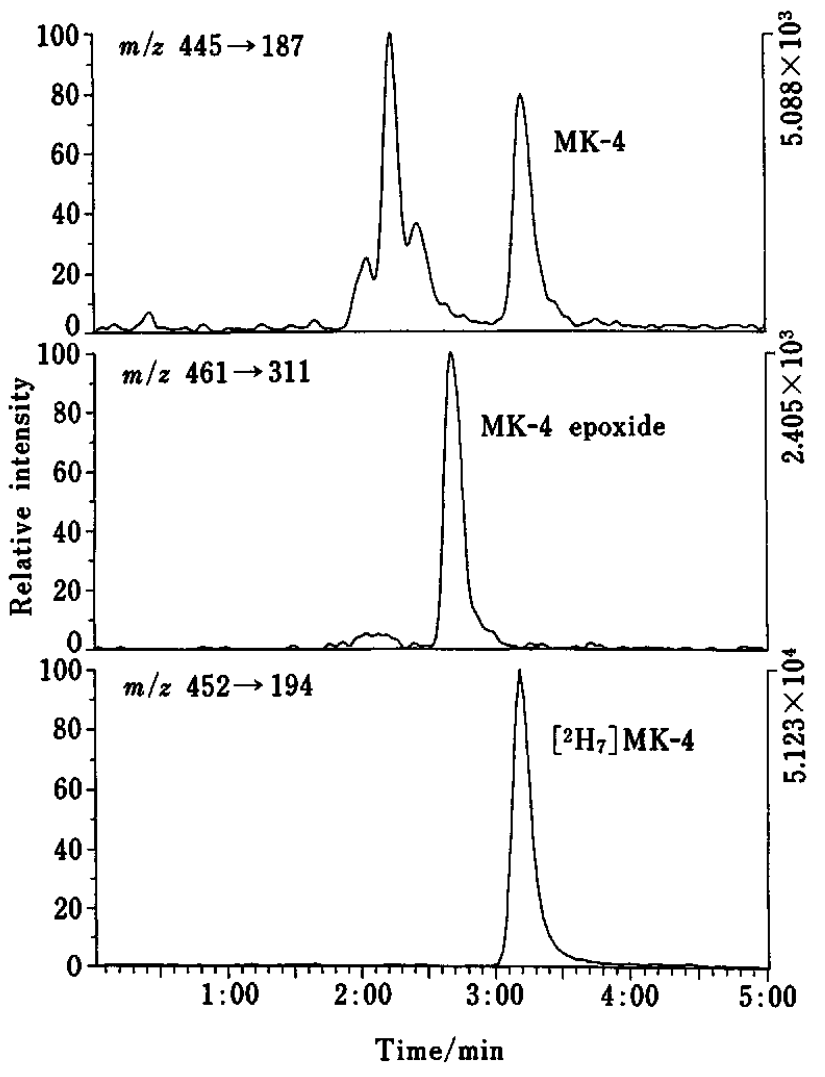

Fig. 7 Typical LC/MS/MS chromatograms for menaquinone- 4 and menaquinone- 4 epoxide extracted from osteoblasts following incubation with menaquinone-4 $(2.5 \times$ $\left.10^{-6} \mathrm{M}\right)$. MK-4: menaquinone-4; MK-4 epoxide: menaquinone-4 epoxide.

following incubation with menaquinone-4. The concentration of menaquinone-4 epoxide increased upon exposure to warfarin (Fig. 7, Table 3). It is therefore suggested that menaquinone- 4 is metabolized to menaquinone-4 epoxide in bone as well as in liver.

In conclusion, an LC/MS/MS method for the simultaneous determination of menaquinone-4 and its metabolite menaquinone- 4 epoxide with minimum pretreatment of biological samples and a short analysis time per sample has been established. The sensitivity, accuracy and precision of the method are satisfactory for measuring the compounds in samples extracted from 
human osteoblasts following incubation with menaquinone-4. The quantification limit of menaquinone-4 in this method is relatively sensitive compared to a previous HPLC method with fluorescence detection (50 pg per injection) ${ }^{14}$ and linear calibration ranges (over $10^{3}$-fold range of concentrations) for both compounds are larger compared to previous studies ${ }^{19-23}$ in which drugs were determined using LC/APCI/MS/MS with calibration ranges of several 10 or $10^{2}$-fold concentrations. Furthermore, we have demonstrated using this method that menaquinone- 4 is metabolized to menaquinone-4 epoxide in bone as well as in liver, indicating the drug has the same role in $\gamma$-carboxylation of vitamin $\mathrm{K}$ dependent proteins in both tissues, even though it has never been reported that vitamin $K$ is metabolized in tissues other than the liver. It is anticipated that this assay method will contribute to clarifying the pharmacological mechanism of action of menaquinone-4 on bone metabolism.

The authors wish to thank Mr. Kazuhiro Kaneko for his technical advice on sample preparation and Dr. Naoki Asakawa for his support during this study.

\section{References}

1. J. W. Suttie, Hepatology, 7, 367 (1987).

2. C. Vermeer, Biochem. J., 266, 625 (1990).

3. M. J. Shearer, Blood Rev., 6, 92 (1992).

4. Y. Koshihara, K. Hoshi and M. Shiraki, J. Clin. Exp. Med., 161, 439 (1992).

5. Y. Koshihara, K. Hoshi, H. Ishibashi and M. Shiraki, Calcif. Tissue Int., in press.

6. Y. Koshihara and K. Hoshi, J. Bone Miner. Res., in press.

7. Y. Akiyama, K. Hara, I. Ohkawa and T. Tajima, Jpn. J. Pharmacol., 62, 145 (1993).
8. H. Orimo, T. Fujita, T. Onomura, T. Inoue, K. Kushida and M. Shiraki, Clin. Eval., 20, 45 (1992).

9. K. Kinoshita, K. Katayama, H. Horie, T. Matsuura, T. Takamatsu, J. Tsutsumi, C. Yamato, T. Fujita, K. Miyao, S. Baba and T. Konishi, Oyo Yakuri-Pharmacometrics, 5, 505 (1971).

10. J. Hirate, I. Horikoshi, J. Watanabe, K. Tadano, C. Yamato and T. Fujita, Oyo Yakuri-Pharmacometrics, 29, 775 (1985).

11. K. Tadano, T. Yuzuriha and Y. Miyake, J. Nutr. Sci. Vitaminol., 35, 393 (1989).

12. K. Tadano, T. Yuzuriha, T. Ikeuchi, T. Sato and T. Fujita, J. Pharmacobio-Dyn., 12, 646 (1989).

13. Y. Sano, K. Tadano, K. Kaneko, K. Kikuchi and T. Yuzuriha, Jpn. Pharmacol. Ther., 23, 2659 (1995).

14. Y. Sano, K. Tadano, K. Kikuchi, K. Kaneko and T. Yuzuriha, J. Nutr. Sci. Vitaminol., 39, 555 (1993).

15. Y. Sano, K. Tadano, K. Kaneko, K. Kikuchi and T. Yuzuriha, Xenobio. Metabol. Dispos., in press.

16. Y. Sano, K. Tadano, K. Kikuchi, K. Kaneko, T. Yuzuriha, T. Kimura and Y. Murayama, Xenobio. Metabol. Dispos., in press.

17. Y. Sano, K. Tadano, K. Kaneko, K. Kikuchi and T. Yuzuriha, J. Nutr. Sci. Vitaminol., 41, 499 (1995).

18. Y. Koshihara, K. Hoshi and M. Shiraki, Biochem. Pharmacol., 46, 1355 (1993).

19. B. Kaye, M. W. H. Clark, N. J. Cussans, P. V. Macrae and D. A. Stopher, Biol. Mass Spectrom., 21, 585 (1992).

20. T. V. Olah, J. D. Gilbert and A. Barrish, J. Pharm. Biomed. Anal., 11, 157 (1993).

21. D. R. Doerge, S. Bajic, L. R. Blankenship, S. W. Preece and M. I. Churchwell, J. Mass Spectrom., 30, 911 (1995).

22. N. G. Knebel, S. R. Sharp and M. J. Madigan, J. Mass Spectrom., 30, 1149 (1995).

23. T. Yasuda, M. Tanaka and K. Iba, J. Mass Spectrom., 31, 879 (1996).

(Received November 8, 1996)

(Accepted December 5, 1996) 